\title{
Experimental rhinovirus infection in volunteers
}

\author{
P.G Bardin, G. Sanderson, B.S. Robinson, S.T. Holgate, D.A.J. Tyrrell
}

Experimental rhinovirus infection in volunteers. P.G Bardin, G. Sanderson, B.S. Robinson, S.T. Holgate, D.A.J. Tyrrell. (CERS Journals Ltd 1996.

ABSTRACT: Experimental viral disease studies in volunteers have clarified many aspects of the pathogenesis of human viral disease. Recently, interest has focused on rhinovirus-associated asthma exacerbations, and new volunteer studies have suggested that airway responsiveness (AR) is enhanced during a cold. For scientific, ethical and safety reasons, it is important to use validated methods for the preparation of a virus inoculum and that the particular virological characteristics and host responses should not be altered. We have prepared a new human rhinovirus (HRV) inoculum using recent guidelines and assessed whether disease characteristics (for example, severity of colds or changes in AR) were retained.

Studies were conducted in 25 clinically healthy volunteers using a validated HRV inoculum in the first 17 and a new inoculum in the subsequent eight subjects. Severity of cold symptoms, nasal wash albumin levels and airway responsiveness were measured, and the new inoculum was prepared from nasal washes obtained during the cold. The new inoculum was tested using standard virological and serological techniques, as well as a polymerase chain reaction for Mycoplasma pneumoniae.

No contaminating viruses or organisms were detected and the methods suggested were workable. Good clinical colds developed in 20 of the 25 subjects and median symptom scores were similar in the validated and new inoculum groups (18 and 17.5 , respectively; $p=0.19)$. All subjects shed virus, and there were no differences noted in viral culture scores, nasal wash albumin and rates of seroconversion in the two groups. Although airway responsiveness increased in both groups $(p=0.02$ and $p=0.05)$, the degree of change was similar.

We have performed experimental rhinovirus infection studies and demonstrated similar clinical disease in two inoculum groups. Amplified airway responsiveness was induced; continuing studies will define the mechanisms and suggest modes of treatment.

Eur Respir J., 1996, 9, 2250-2255.
Immunopharmacology Group, University Medicine, Southampton General Hospital, Southampton, UK.

Correspondence: P.G. Bardin

Stellenbosch University Pulmonary Unit for Research (SUPUR)

Dept of Internal Medicine

University of Stellenbosch

PO Box 19063

Tygerberg 7505

Cape Town

South Africa

Keywords: Common cold

infections

rhinovirus

volunteer studies

Received: March 121996

Accepted after revision August 111996

PGB was supported by a postdoctoral fellowship from the South African MRC. Studies were supported by a grant from the British Lung Foundation and by the Department of Internal Medicine, University of Stellenbosch, Cape Town, South Africa.
Experimental disease studies in volunteers have been valuable instruments to clarify important aspects of the pathogenesis of viral diseases and associated lung complications in humans. Such studies have been particularly useful in human rhinoviral (HRV) illness, the most frequent cause of the common cold. Because recent epidemiological studies have suggested that HRV may be associated with the majority of exacerbations of asthma [1], interest has been focused on this particular group of viruses, and this has prompted new human volunteer studies to examine this observation in more depth. Recently, experimental HRV cold studies in atopic individuals have demonstrated new late asthmatic reactions to allergen provocation in association with infection [2], underscoring the value of examining in vivo responses to viral disease. Rhinovirus serotype 16 infection has consistently induced changes in airway reactivity in volunteers; it is one of more than 100 rhinovirus serotypes causing the majority of common colds in the community.

Early [3-5] and more recent [6, 7] human volunteer studies of HRV colds have clarified important aspects relating to virus transmission, infectivity, culture, serological responses and immunity in normal subjects. All the studies used safety-tested inocula prepared in-house using guidelines suggested by KNIGHT [8] in 1963. Some workers have used tissue culture fluids containing virus, whilst in some centres (including the Common Cold Unit, Salisbury, UK) the virus used was obtained as a nasal washing from a volunteer or group of volunteers living in isolation [9]. The methods used for safety-testing at that time reflected current knowledge and practice and have developed greatly over the subsequent 40 yrs. Recently, updated recommendations were suggested in a consensus report from Gwaltney et al. [10].

The consequences of HRV infection on the lower respiratory tract have not been clarified [11]. Amplified airway responsiveness (AR) was induced by HRV serotype 16 [2], but not by HRV 39 [7, 12] or HRV 2 [13], suggesting that the serotype may be critical. Using HRV 16 in our studies, we have sought similar increases in AR. We have also compared changes in AR in a validated and a new inoculum (derived from the former) to seek additional evidence that viral-host characteristics were 
preserved. Finally, we have taken the opportunity to assess the new guidelines for preparation of an inoculum [10], to assist groups planning to establish research studies.

\section{Methods}

\section{Patients and study design}

The study was conducted in 25 (17 atopic) nonsmoking, clinically healthy volunteers, who did not have neutralizing antibody to HRV 16 in their serum. An inoculum of HRV 16 (a kind gift from E. Dick and W. Busse) was used in the first 17 subjects, and a newly prepared inoculum was used in the subsequent eight volunteers. The studies were approved by the Southampton and District Ethics Committee.

Subjects were studied in three phases, having established absence of neutralizing antibody to HRV 16. Phase 1: baseline observations were conducted 2 days prior to viral inoculation and included serum for repeat of neutralizing antibody and nasal washing for viral culture. Phase 2: this took place in the Infectious Diseases Unit (IDU) at Southampton General Hospital, where subjects were isolated for 7 days during the experimental HRV 16 infection. Following HRV 16 inoculation, subjects had daily nasal washes, assessment of symptoms and repeat serology. Phase 3: all the above investigations were repeated at Weeks 8-10.

\section{Measurement of neutralizing antibodies}

This was undertaken on serum from all volunteers before admission to the study (baseline) as well as on the day prior to inoculation with HRV 16, on Day 5 of experimental disease and, finally, 8-10 weeks later, as described previously [14]

\section{Symptom assessment}

All subjects completed a validated questionnaire daily for 2 weeks prior to, during and for 8-10 weeks after HRV 16 infection. Symptoms were graded 0-3 according to severity, and the criteria for a cold were based on those proposed by JACKSON et al. [15]. Clinical illness was considered present if a volunteer had a minimum cumulative symptom score of 14 over a 4 day period, and had a subjective impression that he had a cold, or if rhinorrhoea was present on at least 3 of the 5 days of observation. Any baseline rhinitic symptoms for 4 days prior to inoculation were subtracted from new cold symptoms.

\section{Nasal lavage and confirmation of viral infection}

Nasal washing was undertaken by instilling $5 \mathrm{~mL}$ of prewarmed Hank's balanced salt solution (HBSS) with $0.5 \%$ gelatin into each nostril, holding for $5 \mathrm{~s}$ and then expelling into a sterile Petri dish. Samples were divided into aliquots and frozen at $-70^{\circ} \mathrm{C}$ until analysed.
Nasal washes were inoculated into Ohio Helen-Lake (HeLa) cell cultures, and HRV infection was diagnosed by means of the typical cytopathic effects (CPE) on light microscopy. Cultures were examined daily and regarded as negative if they showed no CPE after two passes in cell culture. The presence of virus in nasal washes was determined by culture on HeLa cells, and confirmed by neutralization using HRV 16 specific antiserum (ATCC, Cat. No. V105-501-558; Bethesda, MD, USA). Positive infection was established by at least one identified viral isolate and/or a fourfold rise in titre of neutralizing antibody in some cases. Because conventional quantification of viral shedding is difficult, expensive and time-consuming, a scoring procedure for viral culture was devised to reflect the amount of virus shed. Daily nasal washes were cultured and given CPE scores (range 0-4); the scores were then combined for the 3 day period of lavage collection, with a maximum cumulative score of $12(3 \times 4)$. In early experiments, this procedure gave a good indication of viral shedding and paralleled conventional titration measures; titrations on all nasal washes were, thus, not performed.

\section{Albumin measurement}

Measurements on coded samples were performed by rocket immunoelectrophoresis. Sensitivity of the assay was $1 \mu \mathrm{g} \cdot \mathrm{mL}^{-1}$ and the coefficient of variation of repeated measurements was $<5 \%$ [14].

\section{Measurement of airway responsiveness}

Histamine bronchoprovocation was undertaken at baseline, on Day 5 after infection and at 8-10 weeks using a standard bronchial provocation technique, adapted from the method of CHAI et al. [16]. Doubling concentrations of histamine $\left(0.03-32.0 \mathrm{mg} \cdot \mathrm{mL}^{-1}\right)$ were administered via an Inspiron nebulizer until forced expiratory volume in one second (FEV1) fell by more than $20 \%$ of postsaline values. The provocative concentration of histamine producing a $20 \%$ fall in FEV1 (PC20) was derived by linear interpolation of the log-concentration response curve. In subjects in whom a PC20 could not be measured using the highest concentration, it was calculated by extrapolation for one doubling dilution beyond the highest concentration administered [17].

\section{Nasal inoculation with virus}

Nasal inoculation was performed using HRV 16 suspension as described previously [14]. The HRV16 inoculum obtained from W. Busse and E. Dick was used to inoculate the first 17 volunteers who participated in studies. Thereafter, a new inoculum was prepared and used to induce colds in the subsequent eight subjects.

\section{Preparation of HRV 16 inoculum}

Viral shedding (as assessed by viral cultures) was evaluated in the first 17 volunteers, and seven subjects 
were chosen who had consistently produced high levels of HRV 16 in nasal washes. In addition, they did not belong to any high-risk groups-for human immunodeficiency virus (HIV) infection [18]. The nasal washes were divided into two pools and both pools examined for the presence of viral and other pathogens.

\section{Virological screening}

Cultures. The two pools were filtered separately (Nalgene filters, catalogue No. 163-00Z0; Nalge Co., Rochester). Tubes of Oxoid nutrient broth, inoculated with the final drops from each filter and removed to a $37^{\circ} \mathrm{C}$ incubator were observed for growth of aerobes. Filtered inoculum (Pool 1 (P1) and Pool 2 (P2)) was divided into aliquots and vials of both pools were screened.

Inocula P1 and P2 were cultured on HEp-2 cells (for respiratory syncytial virus and coxsackievirus B) and after neutralization with Type 16 rhinovirus antiserum on MRC-5 (for adenoviruses, rhinoviruses, echoviruses and some coxsackievirus A); tissue culture fluids were passaged twice in tests for rhinoviruses. Finally, P1 and P2 were tested on LLC-MK ${ }_{2}$ cells, which were stained by immunofluorescence and observed for influenza or parainfluenza viruses. Inocula were also tested for growth of Haemophilus influenzae and Streptococcus pneumoniae.

Tubes of Oxoid mycoplasma broth were inoculated (with a positive clinical sample as control) and incubated at $37^{\circ} \mathrm{C}$ for growth of Mycoplasma pneumoniae over a period of 3 months.

Animal inoculation. Three litters of suckling mice were inoculated intracerebrally $(20 \mu \mathrm{L})$, intraperitoneally $(50$ $\mu \mathrm{L})$ and subcutaneously $(30 \mu \mathrm{L})$ within $24 \mathrm{~h}$ of birth. The mice were then monitored daily for 2 weeks for any signs of Group A or B coxsackievirus infection, manifested by paralysis.

Mycoplasma pneumoniae screening. A sensitive polymerase chain reaction (PCR) developed by NARITA et al. [19], was used to exclude the presence of M. pneumoniae. A reference preparation of $M$. Pneumoniae (Ref. No. 1011906) was obtained (Public Health Laboratory Service, London, UK), and a dilution series tested to confirm the above sensitivity; both the inocula were also spiked with approximately 10-50 M. pneumoniae organisms to exclude the presence of PCR inhibitors. Product of the PCR was run on 3\% agarose gel (FMC Bioproducts, Rockland, USA), stained with ethidium bromide and visualized under ultra violet (UV) light.

Donor serology. Serum was obtained from the seven subjects who contributed to the pools at the following times: 1) 2 weeks prior to experimental colds; 2) 6 weeks after HRV 16 colds; and 3) 6-9 months after the first sample (for HIV testing). The following infections were sought employing serological techniques: Legionella fluorescent antibody test (FAT); M. pneumoniae complement fixation test (CFT); psittacosis CFT; Q fever CFT; influenza A and B CFT; respiratory syncytial virus CFT; Adenovirus CFT; mumps virus CFT; measles virus CFT; cytomegalovirus (CMV) CFT; herpes simplex CFT; varicella zoster CFT; hepatitis B antigen screen; hepatitis $\mathrm{C}$ immunoglobulin $\mathrm{G}(\mathrm{IgG})$; Treponema pallidum haemagglutination (TPHA) and HIV antibody screen.

Other tests. Skin tests for tuberculosis with a purified protein derivative (PPD) were performed in 6 out of 7 subjects and a chest radiograph was obtained in all volunteers 1-2 months after their HRV 16 cold.

\section{Statistical analysis}

Symptom scores, CPE scores, nasal wash albumin levels, changes in airway responsiveness and altered serology in the two inoculation groups were compared by Mann-Whitney U-test. A p-value equal to or less than 0.05 was considered significant.

\section{Results}

Screening of HRV 16 inoculum

\section{Virological screening}

Cultures. No fungi were isolated on culture media, and after neutralization of HRV 16 no viruses were isolated in MRC-5 or HEp-2 cells from either P1 or P2 pooled nasal washes. Following neutralization of HRV 16, no rhinoviruses or other viruses (for example, adenovirus) were isolated on Ohio HeLa; also no viruses were isolated on LLC-MK 2 cells.

Animal inoculation. The three litters of suckling mice survived 2 weeks and were healthy with no signs of paralysis. One mouse died at an early stage on Day 2, presumably from injury sustained during intracerebral inoculation.

Mycoplasma pneumoniae. Growth of M. pneumoniae was only demonstrated in the medium which had been inoculated with a positive control sample. The remaining tubes inoculated with P1 and P2 were completely clear for up to 3 months.

Sensitivity of the PCR for M. pneumoniae detection was 10-50 organisms as reported previously [19], and PCR product of the expected number of base pairs was detected in both inocula that had been spiked with the organism, confirming the absence of PCR inhibitors. No evidence for the presence of $M$. pneumoniae was found in the two potential inocula after PCR.

Donor serology. All testing was negative with three exceptions, and no rises in serum antibody to any of the pathogens tested were detected. For the three exceptions, fourfold increases in titre after experimental HRV infection (as compared to before) were noted to measles virus, adenovirus and cytomegalovirus, respectively; this was considered to be of uncertain significance and not reflecting clinical disease. Virological testing of nasal washes yielded no positive cultures for adenovirus and 
Table. 1. - Baseline data and response to infection in 25 subjects with HRV experimental infection

\begin{tabular}{|c|c|c|c|c|c|}
\hline $\begin{array}{l}\text { Subject } \\
\text { No. } \\
\end{array}$ & $\begin{array}{l}\text { Age } \\
\text { yrs }\end{array}$ & $\begin{array}{l}\text { Cold symptom } \\
\text { score }\end{array}$ & $\begin{array}{l}\text { Viral culture } \\
\text { CPE score } \\
\end{array}$ & Seroconversion* & $\begin{array}{c}\text { Nasal albumin } \\
\text { changes (D5-D2) }\end{array}$ \\
\hline \multicolumn{6}{|c|}{ Validated HRV inoculum } \\
\hline 1 & 50 & 5 & 12 & $\times 16$ & -90 \\
\hline 2 & 45 & 7 & 12 & $\times 2$ & -3 \\
\hline 3 & 45 & 24 & 12 & ND & ND \\
\hline 4 & 20 & 10 & 4 & 0 & -3 \\
\hline 5 & 21 & 28 & 12 & $\times 8$ & +71.5 \\
\hline 6 & 20 & 36 & 12 & $\times 8$ & +144.5 \\
\hline 7 & 29 & 15 & 12 & $\times 4$ & -1 \\
\hline 8 & 49 & 6 & 12 & $\times 2$ & -14 \\
\hline 9 & 24 & 15 & 10 & ND & ND \\
\hline 10 & 43 & 29 & 10 & $\times 64$ & +294 \\
\hline 11 & 21 & 13 & 10 & $\times 32$ & +74.5 \\
\hline 12 & 47 & 16 & 8 & $\times 2$ & +8 \\
\hline 13 & 33 & 36 & 4 & 0 & +20 \\
\hline 14 & 32 & 20 & 8 & $\times 32$ & +195 \\
\hline 15 & 27 & 24 & 12 & $\times 4$ & +12 \\
\hline 16 & 26 & 18 & 6 & 0 & +14.5 \\
\hline 17 & 22 & 24 & 6 & $\times 4$ & -40 \\
\hline Median & 32 & 18 & 10 & $\times 4$ & 45 \\
\hline \multicolumn{6}{|c|}{ New HRV inoculum } \\
\hline 1 & 20 & 25 & 2 & 0 & +67 \\
\hline 2 & 22 & 24 & 5 & $\times 32$ & +400 \\
\hline 3 & 23 & 24 & 12 & $\times 4$ & 0 \\
\hline 4 & 26 & 18 & 12 & $\times 16$ & +25 \\
\hline 5 & 22 & 12 & 11 & $\times 64$ & ND \\
\hline 6 & 22 & 14 & 11 & $\times 64$ & ND \\
\hline 7 & 35 & 17 & ND & ND & ND \\
\hline 8 & 28 & 12 & ND & ND & ND \\
\hline Median & 22 & 17.5 & 8.5 & $\times 16$ & 46 \\
\hline
\end{tabular}

+: cumulative score over 3 days of nasal washes; *: expressed as fold difference from baseline. HRV: human rhinovirus; CPE: cytopathic effects; D5: Day 5 nasal washing (during cold); D2: Day 2: before cold; ND: not determined.

no clinical history or other parameters were identified that would be consistent with clinical infection by any of the three pathogens. Tests for HIV were repeated after 6-8 months to eliminate the possibility of false negative results in the "window" period, and were consistently negative.

Other tests. No abnormalities were noted on chest radiographs obtained from volunteers. Weakly positive PPD reactions were observed in 3 out of 6 subjects, all of whom had received bacille Calmette-Guérin (BCG) vaccination as children.

Patients, response to infection and comparison of inocula

A total of 64 volunteers were screened for the presence of serum neutralizing antibodies; 33 (52\%) had antibodies present and nine were unsuitable for other reasons. At present, 25 volunteers have participated in studies and no subjects have terminated their participation prematurely. Baseline characteristics of the group are shown in table 1.

A clinical cold developed in 20 out of 25 subjects as evaluated by symptom scores (table 1). Subjects Nos. $1,2,4,8$ and 11 were negative by scoring; they all had positive viral cultures and/or significant rises in neutralizing antibodies. Median cold symptom score was
18 in the first group of volunteers (range 5-36) and 17.5 (range 12-25) in the later (new inoculum) volunteers. Cold symptom scores obtained from the two groups of subjects were not significantly different $(p=0.19)$, nor was there a difference noted between normal and atopic volunteers or in different age groups.

Rhinoviral culture was positive in all subjects (median culture score 10.5). No differences were noted for this and other variables (viral culture scores, changes in nasal albumin levels, seroconversion) between the first 17 subjects (validated inoculum) and the last eight subjects (new inoculum) entered into the study (table 1).

Neutralizing antibodies to HRV 16 were present in fourfold or higher titre in 9 out of 15 subjects in the validated inoculum group and in 5 out of 6 subjects in the new inoculum group. Again, no difference was found between the inoculum that was used and antibody rises after HRV 16 infection.

\section{Changes in airway responsiveness}

Significant increases in AR were noted both in the validated and new inoculum groups of volunteers (fig. 1) during a cold induced by HRV $16(\mathrm{p}=0.02$ and $\mathrm{p}=0.05$, respectively). Median $\mathrm{PC} 20$ was $23.7 \mathrm{mg} \cdot \mathrm{mL}^{-1}$ before the cold and $14.3 \mathrm{mg} \cdot \mathrm{mL}^{-1}$ during the cold in the validated inoculum group. These values were 32 and 16.8 $\mathrm{mg} \cdot \mathrm{mL}^{-1}$, respectively, for the new inoculum group. 

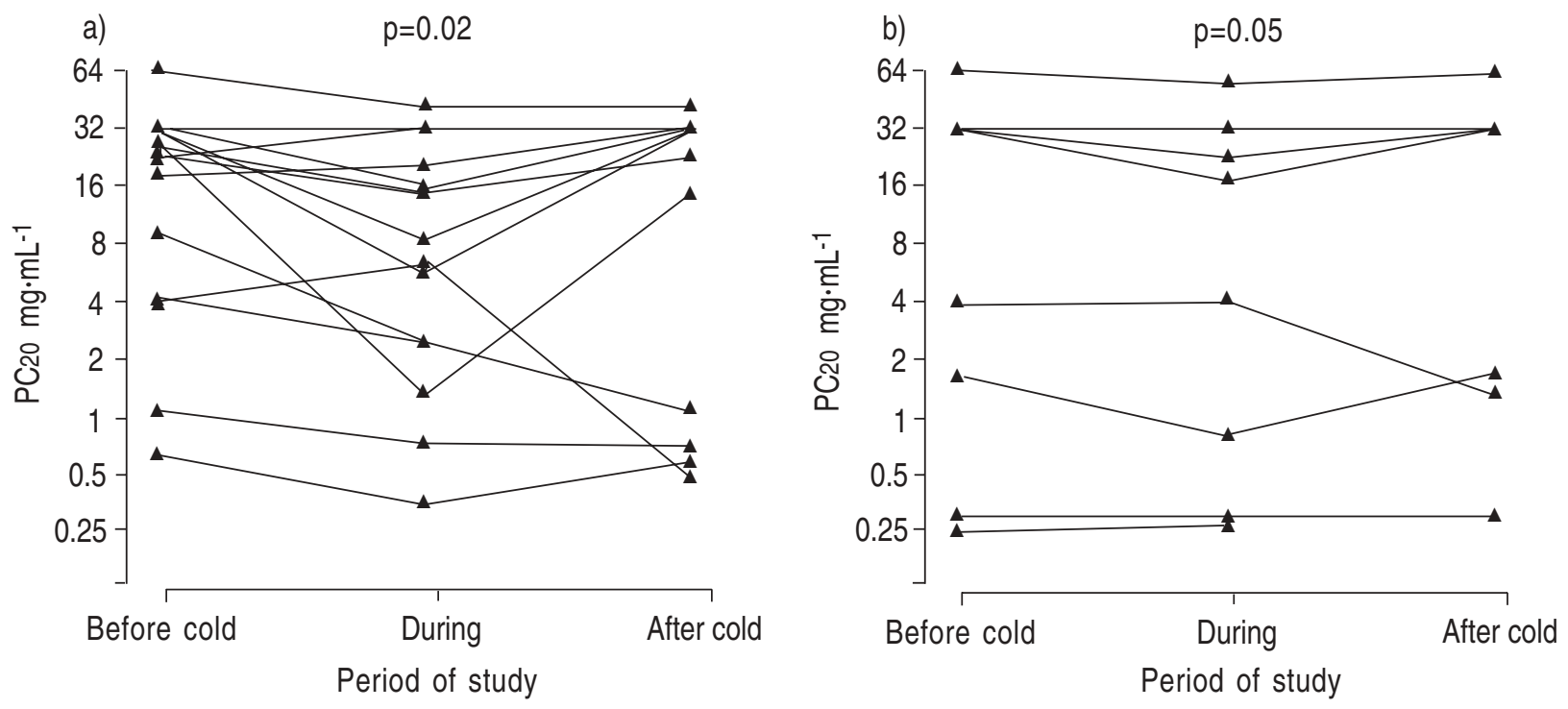

Fig. 1. - Changes in airway reactivity during HRV colds in the two groups of volunteers: a) validated inoculum; b) new inoculum. Amplified reactivity was demonstrated in both groups, but there was no difference in the degree of change between the groups. HRV: human rhinovirus; PC20: provocative concentration of histamine producing a $20 \%$ fall in forced expiratory volume in one second.

Comparison of the degree of change in AR in each group did not reveal any difference $(p=0.3)$.

Atopic subjects demonstrated amplified AR during a cold (mean PC20 $16.3 \mathrm{mg} \cdot \mathrm{mL}^{-1}$ before and $8.7 \mathrm{mg} \cdot \mathrm{mL}^{-1}$ during cold; $\mathrm{p}<0.05$ ), but this was not present in normal individuals (32.6 versus $30.8 \mathrm{mg} \cdot \mathrm{mL}^{-1}$; $\mathrm{p}=0.11$ ). There was no association between age and changes in AR; however, too few patients over 45 yrs of age were studied for any good comparisons.

\section{Discussion}

We have induced HRV 16 infections in two volunteer groups, the second inoculum being derived from nasal washes obtained from volunteers who had a cold induced by the first inoculum. Safety-testing based on new recommendations was performed and did not yield any contaminating viruses or organisms. Clinical disease as measured by symptom scores and AR, infection as reflected by viral shedding and serological responses were identical in both inoculation groups. The methods employed yielded results suggesting that it is feasible to use infectious material to produce inoculum and so develop human volunteer studies of HRV infection.

Over the previous four decades, large numbers of volunteers have been safely infected with respiratory viruses, particularly HRV [4-10]. In recent years, a number of new transmissible infectious agents have been discovered and there have been technological advances which have improved methods for their detection. Updated recommendations for safety-testing have been published [10], concentrating on inocula prepared in cell-culture (usually human embryonic lung cells). We have followed these guidelines, but have varied some investigations based on local conditions and subject characteristics and applied them to an inoculum prepared directly from nasal washes; this was not passed in cultured cells because of the changes in infectivity and other virus-specific characteristics sometimes detected after passage [9]. The period between initial evaluation and retesting for HIV antibody status was set at 6-9 months because this was judged to be an adequate period. In addition, we ensured that none of the volunteers belonged to any of the recognized high-risk groups [18].

Culture of $M$. pneumoniae may be insensitive, and we used a PCR yielding a high detection sensitivity [19]. The presence of PCR inhibitors in nasal washes was excluded using a reference strain of the organism as a positive control. We did not attempt to culture M. tuberculosis as recommended, because of its very low prevalence in our geographical area and because no chest radiograph abnormalities suggesting tuberculosis were noted in any subject. The fourfold increases in CMV, measles and adenovirus titres noted in three volunteers were of uncertain significance. No clinical history of infection immediately prior to study could be obtained and adenovirus was not cultured. Although testing for corona virus was not performed in this study, serological testing is recommended to exclude another important cause of common colds.

The inocula were compared in two groups: an initial group of 17 volunteers (validated inoculum) and a subsequent group of eight subjects (new inoculum). The clinical severity of the colds as measured by symptom scores was identical, as was viral shedding assessed by CPE scores. Importantly, the new inoculum induced significant increases in AR of a degree similar to the initial preparation. This indicates that our previous failure to induce enhanced AR [13] was not due to using nasal washings, but does not indicate whether it was due to the different virus serotype used or the means of administration (spray as well as nasal drops). Nasal wash albumin levels have been shown to accurately reflect the severity of a cold [20], and again identified no differences between the two inoculation groups. Finally, seroconversion after infection was similar in the two groups. Although small differences may not have been detected because of the smaller number of subjects in the second group, it is clear that the clinical and laboratory 
results were very similar in the two volunteer groups. The difference in severity of colds in atopic and normal individuals was noted, as reflected by detectable increases in AR in the atopic group only. This was not demonstrable for symptom scores unless stratification was carried out for baseline antibody levels [14]. The mechanisms implicated remain to be defined.

In summary, safety-screened inocula were prepared from pooled nasal washes and compared to colds produced by a first inoculum. Testing revealed no contamination of pooled nasal washes and no differences were noted between severity of clinical colds or severity of infection in two groups of subjects. Amplified airway responsiveness was induced by human rhinovirus and further volunteer studies employing similar protocols will permit investigation of the mechanisms and suggest new avenues for treatment.

Acknowledgements: The authors thank all volunteers who helped so generously, as well as M. Dorward, S. O'Toole and G. Smith for dedicated patient care.

\section{References}

1. Johnston S, Pattemore P, Smith S, et al. Role of virus infections in exacerbations in children with recurrent wheeze or cough (Abstract). Thorax 1993; 48: 1055.

2. Lemanske RF Jr, Dick EC, Swenson CA, Vrtis RF, Busse WW. Rhinovirus upper respiratory infection increases airway hyperreactivity and late asthmatic reactions. J Clin Invest 1989; 83: 1-10

3. Couch RB, Cate TR, Douglas RG Jr, Gerone PJ, Knight $\mathrm{V}$. Effect of route of inoculation on experimental respiratory viral disease in volunteers and evidence for airborne transmission. Bacteriol Rev 1966; 30: 517-529.

4. Cate TR, Couch RB, Fleet WF, Griffith WR, Gerone PJ, Knight V. Production of tracheobronchitis in volunteers with rhinovirus in a small-particle aerosol. Am J Epidemiol 1965; 81: 95-105.

5. Mufson MA, Ludwig WM, James HD Jr, et al. Effect of neutralizing antibody on experimental rhinovirus infection. Clin Sci 1963; 186: 578-584.

6. Meschievitz CK, Schultz SB, Dick EC. A model for obtaining predictable natural transmission of rhinovirus in human volunteers. J Infect Dis 1984; 150: 195-201.
7. Halperin SA, Eggleston PA, Beasley P, et al. Exacerbations of asthma in adults during experimental rhinovirus infection. Am Rev Respir Dis 1985; 132: 976-980.

8. Knight V. The use of volunteers in medical virology. Prog Med Virol 1964; 6: 1-26.

9. Tyrrell DAJ. The use of volunteers. Am Rev Respir Dis 1963; 88: 128-134.

10. Gwaltney JM Jr, Hendley O, Hayden FG, et al. Updated recommendations for safety-testing of viral inocula used in volunteer experiments of rhinovirus colds. Prog Med Virol 1992; 39: 256-263.

11. Bardin PG, Johnston SL, Pattemore PK. Viruses as precipitants of asthma symptoms. II. Physiology and mechanisms. Clin Exp Allergy 1992; 22: 809-822.

12. Halperin SA, Eggleston PA, Hendley JO, Suratt PM, Groschel DHM, Gwaltney JM. Pathogenesis of lower respiratory tract symptoms in experimental rhinovirus infection. Am Rev Respir Dis 1983; 128: 806-810.

13. Summers QA, Higgins PG, Barrow IG, Tyrrell DAJ, Holgate ST. Bronchial reactivity to inhaled histamine and bradykinin in atopic and nonatopic normal volunteers after experimental rhinovirus infection. Eur Respir $J$ 1992; 5: 313-317.

14. Bardin PG, Fraenkel DJ, Sanderson G, Dorward M, Lau LCK, Johnston SL. Amplified rhinovirus colds in atopic subjects. Clin Exp Allergy 1994; 24: 457-464.

15. Jackson GG, Dowling HF, Spiesman IG, Boand AV. Transmission of the common cold to volunteers under controlled conditions. I. The common cold as a clinical entity. Arch Intern Med 1958; 101: 267-278.

16. Chai H, Froehlich LA, Mathison DA, McLean JA, Rosenthal RR, Sheffer AL. Standardisation of bronchial inhalation challenge procedures. J Allergy Clin Immunol 1975; 56: 322-334.

17. Townley RJ, Hopp RJ. Inhalation methods for the study of airway responsiveness. J Allergy Clin Immunol 1987; 80; 111-124.

18. Bird AG, Gore SM, Burns SM, Duggie JG. Study of infection with HIV and related risk factors in young offenders' institution. BMJ 1993; 307: 228-231.

19. Narita M, Matsuzono Y, Togashi T, Kajii N. DNA diagnosis of central nervous system infection by Mycoplasma pneumoniae. Pediatrics 1992; 90: 250-253.

20. Naclerio RM, Proud D, Lichtenstein LM, et al. Kinins are generated during experimental rhinovirus colds. $J$ Infect Dis 1988; 157: 133-142. 